\title{
Vertical and horizontal processes in the global atmosphere and the maximum entropy production conjecture
}

\author{
S. Pascale ${ }^{1,2}$, J. M. Gregory ${ }^{3,4}$, M. H. P. Ambaum ${ }^{1}$, R. Tailleux ${ }^{1}$, and V. Lucarini ${ }^{2,5}$ \\ ${ }^{1}$ Department of Meteorology, University of Reading, Reading, UK \\ ${ }^{2}$ Meteorologishes Institut, Klimacampus, University of Hamburg, Hamburg, Germany \\ ${ }^{3}$ NCAS-Climate, Meteorology Building, University of Reading, Reading, UK \\ ${ }^{4}$ Met Office Hadley Centre, Exeter, UK \\ ${ }^{5}$ Department of Mathematics and Statistics, University of Reading, Reading, UK
}

Correspondence to: S. Pascale (salvatore.pascale@zmaw.de)

Received: 17 May 2011 - Published in Earth Syst. Dynam. Discuss.: 23 May 2011

Revised: 1 December 2011 - Accepted: 13 January 2012 - Published: 19 January 2012

\begin{abstract}
The objective of this paper is to reconsider the Maximum Entropy Production conjecture (MEP) in the context of a very simple two-dimensional zonal-vertical climate model able to represent the total material entropy production due at the same time to both horizontal and vertical heat fluxes. MEP is applied first to a simple four-box model of climate which accounts for both horizontal and vertical material heat fluxes. It is shown that, under condition of fixed insolation, a MEP solution is found with reasonably realistic temperature and heat fluxes, thus generalising results from independent two-box horizontal or vertical models. It is also shown that the meridional and the vertical entropy production terms are independently involved in the maximisation and thus MEP can be applied to each subsystem with fixed boundary conditions. We then extend the four-box model by increasing its resolution, and compare it with GCM output. A MEP solution is found which is fairly realistic as far as the horizontal large scale organisation of the climate is concerned whereas the vertical structure looks to be unrealistic and presents seriously unstable features. This study suggest that the thermal meridional structure of the atmosphere is predicted fairly well by MEP once the insolation is given but the vertical structure of the atmosphere cannot be predicted satisfactorily by MEP unless constraints are imposed to represent the determination of longwave absorption by water vapour and clouds as a function of the state of the climate. Furthermore an order-of-magnitude estimate of contributions to the material entropy production due to horizontal
\end{abstract}

and vertical processes within the climate system is provided by using two different methods. In both cases we found that approximately $40 \mathrm{~mW} \mathrm{~m}^{-2} \mathrm{~K}^{-1}$ of material entropy production is due to vertical heat transport and $5-7 \mathrm{~mW} \mathrm{~m}^{-2} \mathrm{~K}^{-1}$ to horizontal heat transport.

\section{Introduction}

In 1975 G.W. Paltridge suggested that the Earth's climate structure might be organised in such a way as to maximise its entropy production due to meridional heat transport (MEP Paltridge, 1975). Since then there has been a long and lively debate on MEP. This conjecture, often controversial (Goody, 2007; Caldeira, 2007; Nicolis and Nicolis, 2010), has been mainly tested through simple energy-balance boxmodels (Paltridge, 1975, 1978, 1981; Grassl, 1981; Noda and Tokioka, 1983; Lorenz et al., 2001; Pujol and Fort, 2002; Pujol, 2003; Kleidon, 2004, 2010; Jupp and Cox, 2010; Herbert et al., 2011a) and, in a few cases, general circulation models (Kleidon et al., 2003, 2006; Ito and Kleidon, 2005; Kunz et al., 2008; Pascale et al., 2011b) whereas a rigorous mathematical proof is still missing (Dewar, 2005; Grinstein and Linsker, 2007). Therefore despite some evidence which has built up in geosciences and despite its attraction of offering a beautiful unifying picture for all disequilibrium processes in the Earth system (Kleidon, 2010) and for the several co-related extremal principles known in Fluid 
Dynamics (Malkus, 1954, 1956, 2003; Lorenz, 1960; Busse, 1969, 1970), MEP still has a shadowy theoretical foundation.

Furthermore, the interpretation of MEP still remains an open issue. For almost three decades after its appearance in the climate science community, MEP was seen as a new physical principle, a sort of extension of the second law of thermodynamics to nonequilibrium steady states (Grassl, 2005; Martyushev and Seleznev, 2006). A recent reappraisal of MEP (Dewar, 2009; Dyke and Kleidon, 2010) as an inference algorithm that passively translates physical assumptions into macroscopic predictions (Maximum Entropy or, colloquially, MaxEnt, Jaynes, 1957) gives a quite different interpretation. In this case the disagreement of MEP predictions with observations (or realistic models) means only that the physical assumptions we have used to define the model are either wrong or insufficient. However it has to be noted that in equilibrium thermodynamics there is no room for this duality of principle versus algorithm, as the second law or principle of Thermodynamics has a statistical interpretation and Statistical Mechanics of equilibrium (consistent with MaxEnt, as shown by Jaynes, 1957) offers an algorithm which predicts that behaviour. In non-equilibrium thermodynamics these relationships between macroscopic thermodynamic description and statistical mechanics, and between statistical mechanics and MaxEnt, are not yet well established.

In the meantime in the last decade progress has been made in our knowledge of the entropy budget of the Earth's system and, more generally, of the basic Thermodynamics of the general circulation of the atmosphere and oceans (Lucarini, 2009). The total material entropy production of the climate system has been estimated from general circulation models to be about $50 \mathrm{~mW} \mathrm{~K}^{-1} \mathrm{~m}^{-2}$ and most of it is associated with the hydrological cycle whereas only a small $(10 \%)$ fraction is associated with large scale meridional heat transport (Ambaum, 2010; Fraedrich and Lunkeit, 2008; Pascale et al., 2011a). On the basis of this, recently Lucarini et al. (2011) have questioned the appropriateness of 2-box models as the paradigmatic one used by Lorenz et al. (2001) as a tool to investigate MEP. Given the large difference in the magnitude of the two contributions as well as the different nature of the atmospheric motions (fast small-scale vertical processes such as convection vs. slower large scale meridional heat transport) from which they are generated, substantial difference may be expected when considering MEP. To the authors' knowledge the only studies of MEP taking into account both horizontal and vertical material entropy production are the ones in Noda and Tokioka (1983) and Herbert et al. (2011b). Noda and Tokioka (1983) extended Paltridge's work to a two-dimensional zonal model with prescribed water vapour but variable low, middle and high clouds. However the MEP solutions they found were shown to be very sensitive to the parametrisation of humidity, since either multiple maxima or no maximum at all could be obtained for certain distribution of relative humidity. Herbert et al. (2011b) presented a new formulation of Paltridge's model in which the Net-Exchange Formulation of radiative transfer is employed (Dufresne et al., 2005), the ad hoc convective hypothesis of Paltridge (1978) is avoided and the total material entropy production maximised, but they do not consider the vertical thermal structure of the atmosphere.

The objective of this paper is to reconsider MEP in the context of a very simple two-dimensional zonal-vertical climate model able to represent the total material entropy production due to both horizontal and vertical heat fluxes. First, a simple four-box model with prescribed solar heating is used (Sect. 2) in order to show the relationship between horizontal and vertical entropy production in the maximisation process. Second, we extend the resolution of the model and make a more precise comparison of the MEP solution with a stateof-the-art general circulation model climatology (Sect. 3). The only other paper in which a comparison is made between a MEP solution and a general circulation model (supposed to be well representative of the real climate) is that of Ito and Kleidon (2005), who however use a dry dynamic core with prescribed surface temperature. Consequently only the atmosphere is taken into account, and the material entropy production due to hydrological cycle is not included. Previously, Noda and Tokioka (1983) had considered a twodimensional zonal model (10 latitude zones) dealing with the full material entropy production but made no direct comparison with climatology or more complex model outputs. Finally in Sect. 4.1 we analyse the solutions in terms of vertical/horizontal splitting and provide some new independent estimates which agree with results in Lucarini et al. (2011).

\section{Simple four-box model for material entropy production}

\subsection{The model}

A simple conceptual box-model for climatic entropy production is shown in Fig. 1, where by label 1 we denote the tropical zone and by 2 the extratropical one. Such a model is not meant to be a more involved version of the two-box model described by Lorenz et al. (2001) but rather a minimal conceptual model for material entropy production in a planetary system, as proposed by Lucarini et al. (2011), since it accounts for both horizontal and vertical transport processes. Lucarini et al. (2011) claim that two-box models as in Lorenz et al. (2001), by neglecting vertical processes, do not provide a realistic description of the material entropy production and therefore cannot be used to test MEP. The longwave and shortwave transmissivities of each atmospheric box, $\tau_{1, i}, \tau_{\mathrm{s}, i}, i=1,2$ are prescribed and to be considered parameters of the model. The longwave emissivity is assumed to be $\epsilon_{i}=1-\tau_{1, i}$ according to Kirchhoff's law. Such a model has no albedo-, cloud-, and water vapour- feedback since the albedo and the transmissivities are fixed. As a consequence the net insolation of each box, $I_{1}, I_{2}$, which in 


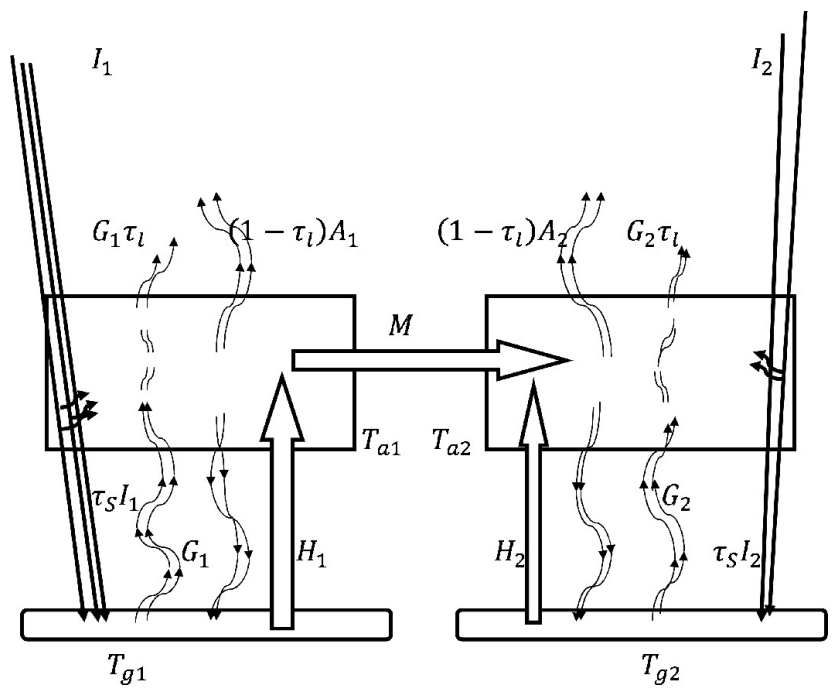

Fig. 1. Simple model for representing the material entropy production of the climate system. The model consists of "tropical" and "high-latitude" surface-atmosphere components which exchange heat via the meridional transport $M$. The surface and the atmosphere interact through the fluxes $H_{1}$ and $H_{2}$ (latent and sensible heat).

general depends on the surface and atmosphere state (surface and planetary albedo), is fixed as well.

The values of $\tau$ (see Table 1) have been worked out from a control run obtained with the FAMOUS AOGCM after defining a "tropical" $\left(30^{\circ} \mathrm{N}-30^{\circ} \mathrm{S}\right)$ and an "extratropical" box. FAMOUS (Jones et al., 2005; Smith et al., 2008), is the lowresolution version of HadCM3 (Gordon et al., 2000; Pope et al., 2000), which has been widely used to simulate present day and future climate and compares well with current general circulation models and observations (Reichler and Kim, 2008). The FAMOUS solution can be considered a relatively good representation of real climatology, as shown in Jones et al. (2005). Therefore in the following we will use it to assess MEP solutions.

The surface interacts with the atmosphere through the vertical fluxes of latent plus sensible heat, $H_{1}$ and $H_{2}$. Atmospheric boxes 1 and 2 also exchange energy due to the horizontal heat flux $M$. The values of the material energy fluxes $H_{1}, H_{2}$ and $M$ are not known a priori in our model since their values depend on dynamical details which are not dealt with in such a simplified model. Assuming the system to be in a steady state, the sum of the heating rates due to radiation and material heat fluxes has to vanish for each box:

$$
\begin{aligned}
& I_{1}\left(1-\tau_{\mathrm{s}, 1}\right)+H_{1}-M+\left(1-\tau_{1,1}\right)\left(G_{1}-2 A_{1}\right)=0 \\
& I_{2}\left(1-\tau_{\mathrm{s}, 2}\right)+H_{2}+M+\left(1-\tau_{1,2}\right)\left(G_{2}-2 A_{2}\right)=0 \\
& \tau_{\mathrm{s}, 1} I_{1}-G_{1}-H_{1}+\left(1-\tau_{1,1}\right) A_{1}=0 \\
& \tau_{\mathrm{s}, 2} I_{2}-G_{2}-H_{2}+\left(1-\tau_{1,2}\right) A_{2}=0
\end{aligned}
$$

Table 1. Parameters used in the box-model shown in Fig. 1. The values have been obtained from a FAMOUS control run after defining a "tropical" box in the GCM with edges at $30^{\circ} \mathrm{N}-30^{\circ} \mathrm{S}$ and an "extra-tropical" box for the complementary polar caps. With this choice the areas of the two boxes are equal. $\tau_{1}$ has been worked out by using the approximation in Eq. (12) and defined for the boxmodel as the total transmissivity of the atmosphere, i.e. the product of $\tau_{1}$ at all atmospheric levels.

\begin{tabular}{lccc}
\hline Quantity & $\begin{array}{c}\text { Tropical } \\
\text { region }\end{array}$ & $\begin{array}{c}\text { Extra-tropical } \\
\text { region }\end{array}$ \\
\hline Insolation $\left(\mathrm{W} \mathrm{m}^{-2}\right)$ & $I$ & 302 & 180 \\
LW transmissivity & $\tau_{1}$ & 0.018 & 0.034 \\
SW transmissivity & $\tau_{\mathrm{s}}$ & 0.70 & 0.62 \\
\hline
\end{tabular}

where $G_{i}=\sigma T_{g, i}^{4}$ and $A_{i}=\sigma T_{\mathrm{a}, i}^{4}, T_{g, 1}$ and $T_{g, 2}$ the surface temperature of zone 1 and $2, T_{\mathrm{a}, 1}$ and $T_{\mathrm{a}, 2}$ the atmospheric temperature of zone 1 and 2, and $\sigma$ is the Stefan-Boltzmann constant. Since we have four equations and seven unknowns $\left(H_{1}, H_{2}, M, T_{g, i}, T_{\mathrm{a}, i}^{4}\right)$, we can express three of them as functions of the remaining four. Therefore from Eqs. (1)-(4) temperatures can be expressed as functions of the heat fluxes:

$$
\begin{aligned}
& T_{\mathrm{a} 1}=\left(\frac{1}{\sigma} \frac{I_{1}\left(1-\tau_{1,1} \tau_{\mathrm{s}, 1}\right)+\tau_{1,1} H_{1}-M}{1-\tau_{1,1}^{2}}\right)^{1 / 4}, \\
& T_{\mathrm{a} 2}=\left(\frac{1}{\sigma} \frac{I_{2}\left(1-\tau_{1,2} \tau_{\mathrm{s}, 2}\right)+\tau_{1,2} H_{2}+M}{1-\tau_{1,2}^{2}}\right)^{1 / 4}, \\
& T_{g 1}=\left(\frac{1}{\sigma} \frac{\left(1+\tau_{\mathrm{s}, 1}\right) I_{1}-H_{1}-M}{1+\tau_{1,1}}\right)^{1 / 4}, \\
& T_{g 2}=\left(\frac{1}{\sigma} \frac{\left(1+\tau_{\mathrm{s}, 2}\right) I_{2}-H_{2}+M}{1+\tau_{1,2}}\right)^{1 / 4} .
\end{aligned}
$$

The material entropy production in the box-model shown in Fig. 1 is generated by three different fluxes $M, H_{1}$ and $\mathrm{H}_{2}$ which carry heat through the temperature differences $T_{\mathrm{a}, 1}-T_{\mathrm{a}, 2}, T_{g, 1}-T_{\mathrm{a}, 1}, T_{g, 2}-T_{\mathrm{a}, 2}$ (i.e. by the fluid response to the radiative forcing) and it reads:

$$
\begin{aligned}
\dot{S}_{\mathrm{mat}} & =M\left(\frac{1}{T_{\mathrm{a} 2}}-\frac{1}{T_{\mathrm{a} 1}}\right)+H_{1}\left(\frac{1}{T_{\mathrm{a} 1}}-\frac{1}{T_{g 1}}\right) \\
& +H_{2}\left(\frac{1}{T_{\mathrm{a} 2}}-\frac{1}{T_{g 2}}\right) .
\end{aligned}
$$

Equation (9) comes from the general expression $\mathbf{F} \cdot \nabla(1 / T)$ for entropy production due to a heat flux $\mathbf{F}$ flowing through a temperature gradient (DeGroot and Mazur, 1984). A very similar formula has also been derived from first principles by Lucarini et al. (2011). $\dot{S}_{\text {mat }}$ is therefore the sum of two 
different contributions: $\dot{S}_{\text {hor }}=M\left(1 / T_{\mathrm{a}, 1}-1 / T_{\mathrm{a}, 2}\right)$ due to the horizontal motions of the atmosphere and $\dot{S}_{\mathrm{ver}}=H_{1}\left(1 / T_{\mathrm{a}, 1}-\right.$ $\left.1 / T_{g, 1}\right)+H_{2}\left(1 / T_{\mathrm{a}, 2}-1 / T_{g, 2}\right)$ due to surface-atmosphere coupling through convective fluxes. In the real climate $\dot{S}_{\text {ver }}$ can be thought of as the entropy production due to the sum of latent and sensible heat fluxes at the surface (Kleidon, 2009) and, to a minor extent, turbulent dissipation of kinetic energy. Lucarini et al. (2011) have shown that $\dot{S}_{\text {hor }}$ is a lower bound of entropy production due to dissipation of kinetic energy.

Material entropy production is therefore a function of $\left(M, H_{1}, H_{2}\right)$ and thus defined in the $\left(M, H_{1}, H_{2}\right)$ space. We do not consider the large entropy production due to irreversible changes in the spectral properties of the radiation (mainly thermalisation of solar radiation) which, as clarified by Ozawa et al. (2003), is unimportant for the dynamics of the climatic fluid.

\subsection{MEP solution}

$\dot{S}_{\text {mat }}$, shown in Figs. 2a-3a, has a unique maximum for $\left(M_{\text {mep }}, H_{1, \text { mep }}, H_{2, \text { mep }}\right) \approx(34.5,113,55) \mathrm{W} \mathrm{m}^{-2}$. Values of energy fluxes, temperatures and entropy production of the MEP solution are summarised in Table 2 and compared with FAMOUS climatology, revealing a certain degree of realism particularly in the atmospheric temperatures and heat fluxes. Surface temperatures are generally lower than FAMOUS climatology and also the material entropy production is considerably underestimated by the MEP solution.

From Fig. $3 b$ we observe that the horizontal component of the material entropy production $\dot{S}_{\text {hor }}$ is quasiindependent of $H_{1}$ and $H_{2}$, because its largest values are placed at $M \approx 30 \mathrm{~W} \mathrm{~m}^{-2} \approx M_{\text {mep }}$ regardless of $H_{1}$ (and $H_{2}$, not shown). Similarly, $\dot{S}_{\text {ver }, 1}$ is maximised by approximately the same $H_{1}$ regardless of $M$, and likewise $\dot{S}_{\text {ver, } 2}$ by $H_{2}$, as can be seen in Fig. 4a-b. $\dot{S}_{\text {ver }}$ (Fig. 5a-b) shows a well defined peak in the $\left(H_{1}, H_{2}\right)$ plane, but has a very weak dependence on the meridional heat transport $M$ (Fig. 5a), unlike $\dot{S}_{\text {mat }}$. There is therefore a kind of "orthogonality" in the material entropy production which allows us to formulate the MEP conjecture for either $\dot{S}_{\text {hor }}$ or $\dot{S}_{\text {ver }}$ separately. This means that, for example, a steady state could be in a state of maximum horizontal entropy production without maximising the total material entropy production.

The main difference between $\dot{S}_{\text {mat }}$ and $\dot{S}_{\text {hor }}, \dot{S}_{\mathrm{ver}, 1}, \dot{S}_{\mathrm{ver}, 2}$ is that only the first one has a unique local maximum whereas the last three exhibit a sort of ridge but no local maxima. Therefore we deduce that MEP can uniquely predict the overall flux structure of this climate model only when the whole material entropy production is taken into account. However the same plots show that if we restrict ourselves to either the atmosphere or to a vertical subsystem surface-atmosphere of our model, and regard the other fluxes external to these as fixed boundary conditions, we retrieve MEP for that particular subsystem.

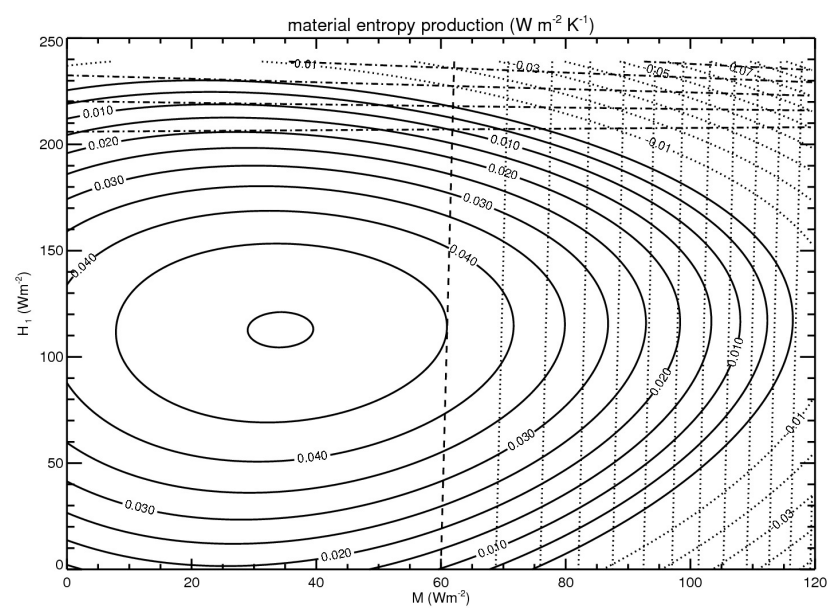

(a)

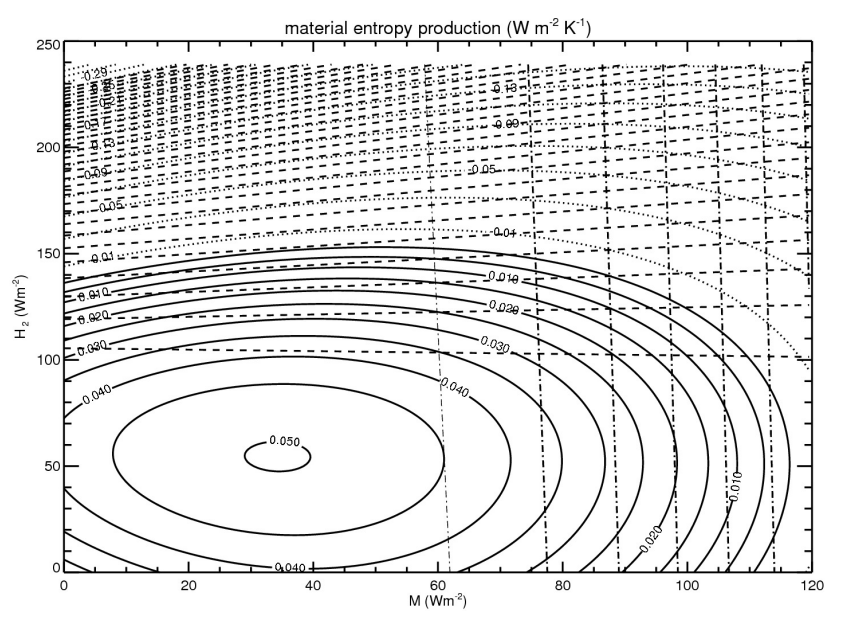

(b)

Fig. 2. (a) $\dot{S}_{\text {mat }}$ in the $\left(M, H_{1}\right)$ plane at $H_{2}=H_{2 \text {,mep }}$ and (b) $\dot{S}_{\text {mat }}$ in the $\left(M, H_{2}\right)$ plane at $H_{2}=H_{1}$,mep. The dashed and dotted lines indicate the portion of the flux-space in which either $\dot{S}_{\text {hor }}$ and $\dot{S}_{\text {ver, } 1}$ $-\dot{S}_{\text {ver }, 2}$ in (b) - are negative (compare with Figs. 3b-5b).

\section{Increasing the resolution of the simple model}

\subsection{Resolution}

In order to obtain a model which is more easily comparable with FAMOUS climatology we refine the spatial resolution of the climate model shown in Fig. 1. We maintain the same physics but increase the number of "boxes" in the meridional (latitude zones) and vertical direction (so the model remains zonal). In particular we consider eleven vertical boxes coincident with FAMOUS atmospheric vertical layers. There are 17 boxes in latitude $\left(11.25^{\circ}\right)$. FAMOUS has a meridional grid spacing of $5^{\circ}$ so its field are regridded by area-averaging in order to match the box-model horizontal resolution. 


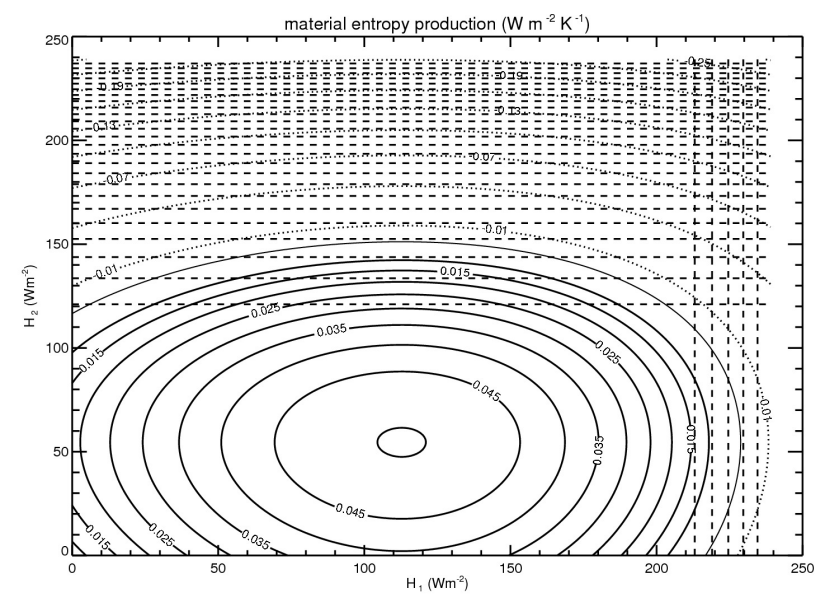

(a)

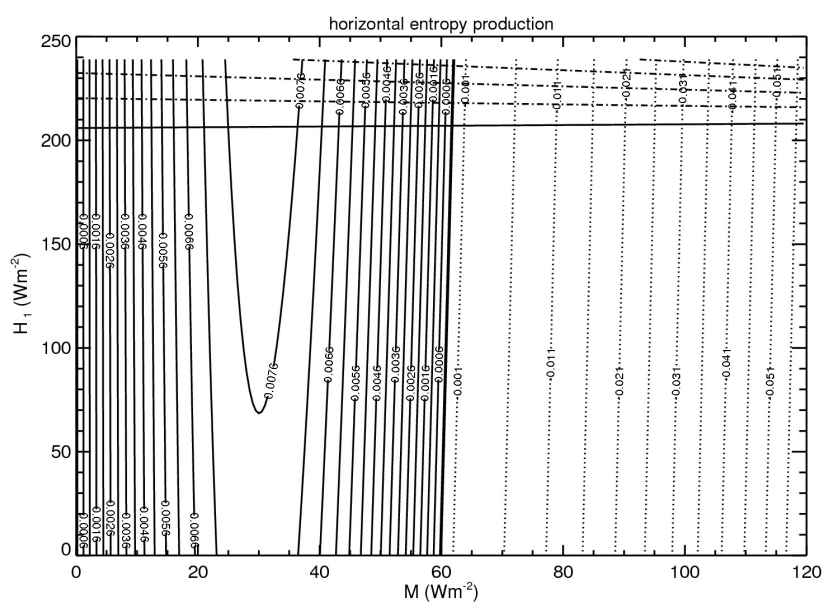

(b)

Fig. 3. (a) $\dot{S}_{\text {mat }}$ in the $\left(H_{1}, H_{2}\right)$ plane at $M=M_{\text {mep }}$ and (b) $\dot{S}_{\text {hor }}$ in $\left(M, H_{1}\right)$ plane at $H_{2}=H_{2}$,mep. The dashed and dotted lines indicate the portion of the flux-space in which either $\dot{S}_{\text {hor }}$ or $\dot{S}_{\text {ver }}$ i.e. $\dot{S}_{\text {ver }, 1}$ in (a) or $\dot{S}_{\text {ver }, 2}$ in (b) - are negative.

The interior of the ocean is neglected. Although the material entropy production due to the small-scale eddy turbulence $\left(\sim 1 \mathrm{~mW} \mathrm{~m}^{-2} \mathrm{~K}^{-1}\right)$ is negligible when compared to the material entropy production of the whole climate system $\left(\sim 50 \mathrm{~mW} \mathrm{~m}^{-2} \mathrm{~K}^{-1}\right.$, see Shimokawa and Ozawa, 2001; Pascale et al., 2011a), the ocean meridional heat transport is of the same order of magnitude of the atmospheric one (Paltridge, 1978; Trenberth et al., 2009). Therefore the consequence of this omission may be an enhancement of the atmospheric meridional heat transport and consequently a reduction of the surface meridional gradient.

\subsection{Radiative parametrisation}

The shortwave and longwave optical properties of the atmosphere (the analogue quantities of $\tau_{\mathrm{s}, i}, \tau_{1, i}$ in Fig. 1) are estimated from a 30-year FAMOUS control run with preindustrial $\mathrm{CO}_{2}$ concentration as follows:

- The solar energy input (shortwave heating rates within the atmosphere, shortwave fluxes at the surface and top of the atmosphere) is fixed and taken from a 30-year time-mean FAMOUS control run (Smith et al., 2008). This ensures fixed "forcing" boundary conditions in full analogy with the four 4-box model considered in Sect. 2.1. It is equivalent to fixing the $\tau_{\mathrm{s}}$ shown in Fig. 1. Fixing the solar (shortwave) input is an assumption as restrictive as fixing the longwave optical properties because in the real climate the shortwave cloud feedback and the sea-ice feedback are very important state-dependent mechanisms which can substantially alter the amount of solar heating received by climate components (see Herbert et al., 2011a for an example of the importance of the ice-albedo feedback in a box-model). However this is a simplifying hypothesis often assumed in the MEP literature (Lorenz et al., 2001; Rodgers, 1976; Murakami and Kitoh, 2005; Ozawa and Ohmura, 1997; Pujol and Fort, 2002; Kleidon et al., 2003, 2006; Kunz et al., 2008).

- The longwave optical properties of the atmosphere (emissivity, transmissivity, scattering) are in general very complex functions of the concentrations of the absorbing gases and aerosols and of the climatic state (pressure, temperature, cloud cover). A fully consistent treatment would thus demand a state-of-the-art radiation model. In order to retain the simplicity of the model discussed in Sect. 2 we make here the drastic assumption of constant emissivity and transmissivity in each grid box. The validity of this simplifying assumption is limited to cases where the predicted temperature distributions are not very apart from the FAMOUS mean state. Furthermore, we neglect the scattering of longwave radiation (which however is accounted for in FAMOUS). This is justified by the high asymmetry factors and the low single scattering albedo for infrared radiation (Edwards and Slingo, 1996), which make absorption dominant over scattering. The main effect of longwave radiation scattering is a reduction of the outgoing longwave radiation of $\sim 2 \mathrm{~W} \mathrm{~m}^{-2}$ (Edwards and Slingo, 1996).

The broad mean transmissivity of each atmospheric box is deduced from the diagnostics of the upwelling and downwelling longwave fluxes (available at each model half level at the interfaces between model layers), say $U(z+1 / 2), D(z+1 / 2)$ (to simplify the notation here we omit the dependence on $x$ and $y$ ). By assuming that each grid-box at the vertical level $z$ of mean 


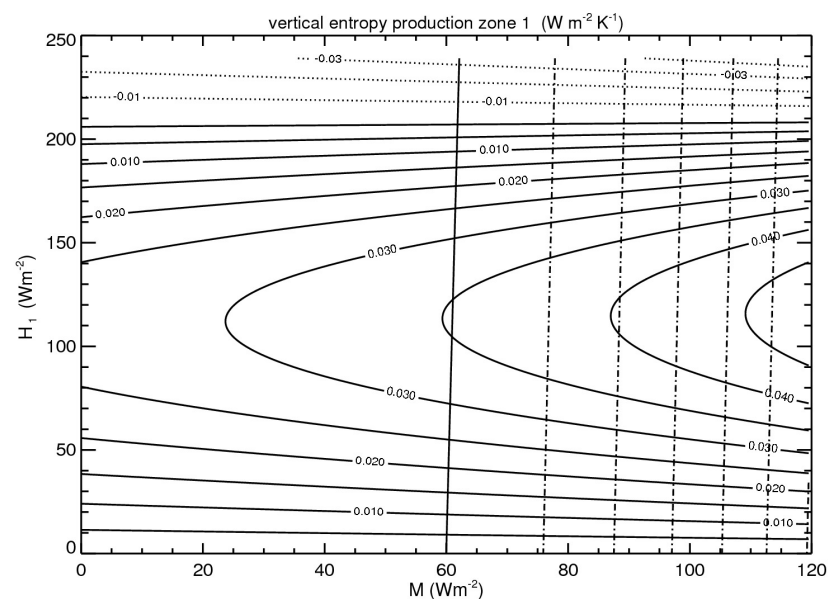

(a)

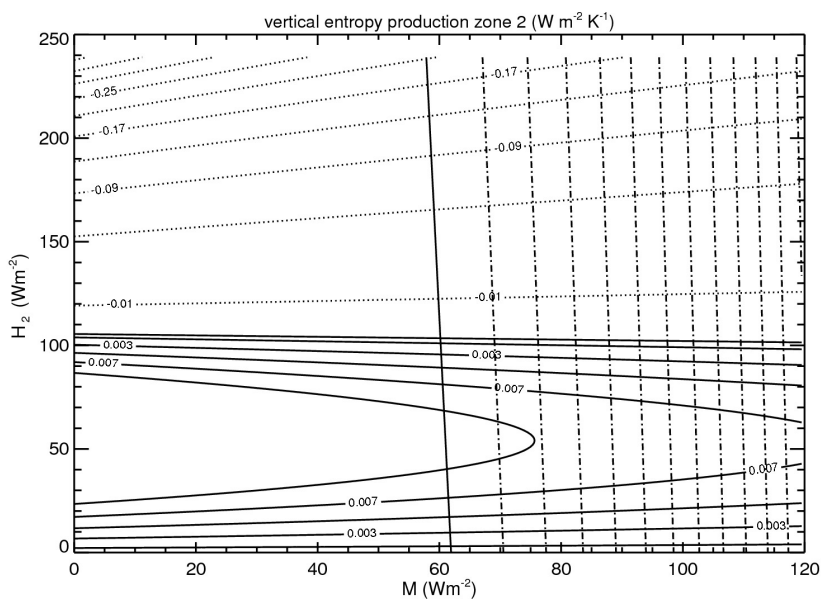

(b)

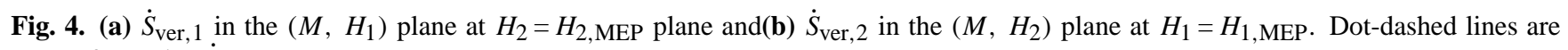
areas of negative $\dot{S}_{\text {hor }}$

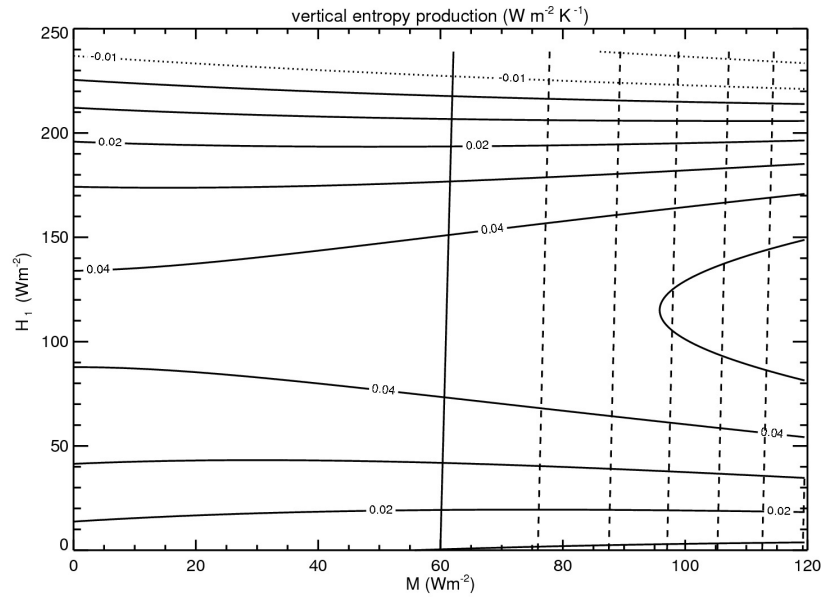

(a)

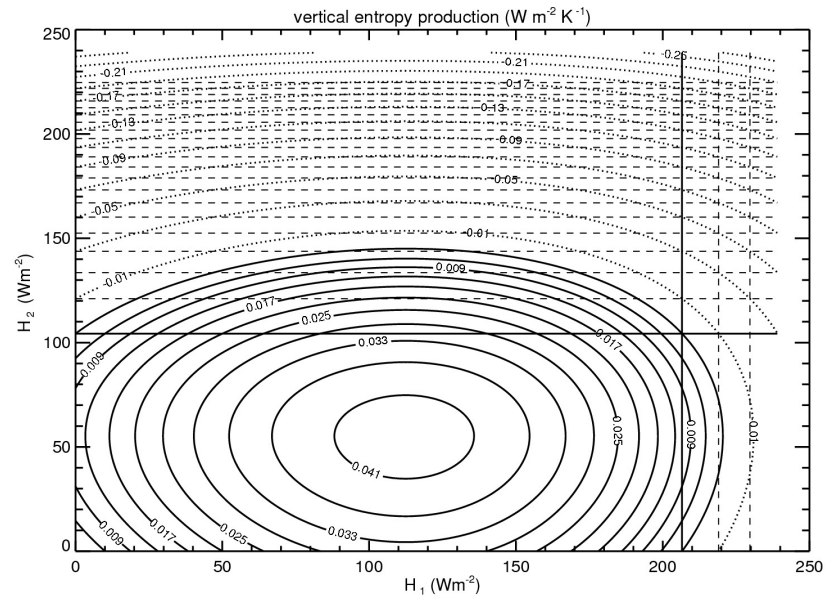

(b)

Fig. 5. (a) $\dot{S}_{\text {ver }}$ in $\left(M, H_{1}\right)$ plane at $H_{2}=H_{2, \text { mep }}$ (dashed lines denote the region of negative $\left.\dot{S}_{\text {hor }}\right)$ and (b) $\dot{S}_{\text {ver }}$ in $\left(H_{1}, H_{2}\right)$ plane at $M=M_{\text {mep }}$ (with areas of negative $\dot{S}_{\mathrm{ver}, 1}$ and $\dot{S}_{\mathrm{ver}, 2}$ overdotted).

transmissivity $\tau_{1}(z)$ emits $e(z)$ upwards and downwards, we must have that:

$U(z+1 / 2)=e(z)+\tau_{1}(z) U(z-1 / 2)$,

$D(z-1 / 2)=e(z)+\tau_{1}(z) D(z+1 / 2)$,

from which the following estimate for $\tau_{1}(z)$ at every model level $z$ is obtained:

$\tau_{1}(z)=\frac{U(z+1 / 2)-D(z-1 / 2)}{U(z-1 / 2)-D(z+1 / 2)}$.

$\tau_{1}(z)$ is shown in Fig. 6. We define the emissivity at each grid-point either as $\epsilon(z) \equiv e(z) / \sigma T(z)^{4}$, by using the energy emissions found in Eq. (10), or as $\epsilon(z)=1-\tau_{1}(z)$, in full analogy with the model in Sect. 2.1. The definitions of $\epsilon(z)$ do not exactly coincide (differences of percents) as we would expect given the very crude approximation implied by Eqs. (10)(11) in which we neglect longwave scattering and spectral dependence (as it is in Edwards and Slingo, 1996). The first definition allows us to match the model entropy budget and the TOA fluxes and therefore should be regarded more as a parametrisation of the box model against climatology. 
Table 2. Values of the variables defining the minimal box-model in Fig. 1 obtained from the maximisation of $\dot{S}_{\text {mat }}\left(M, H_{1}, H_{2}\right)$ and from a FAMOUS control run after defining a "tropical" and "extra-tropical" zone in the GCM with edges at $30^{\circ} \mathrm{N}-30^{\circ} \mathrm{S}$. Values of the total and vertical material entropy productions are averaged over the two zones.

\begin{tabular}{lllrl}
\hline Quantity & & MEP & FAMOUS & Units \\
\hline Vertical heat flux 1 & $H_{1}$ & 113 & 138 & $\mathrm{~W} \mathrm{~m}^{-2}$ \\
Vertical heat flux 2 & $H_{2}$ & 55 & 64 & $\mathrm{~W} \mathrm{~m}^{-2}$ \\
Meridional heat transport & $M$ & 34.5 & 39 & $\mathrm{~W} \mathrm{~m}^{-2}$ \\
Atmospheric temperature 1 & $T_{\mathrm{a}, 1}$ & 261.7 & 258 & $\mathrm{~K}$ \\
Atmospheric temperature 2 & $T_{\mathrm{a}, 2}$ & 247.5 & 247 & $\mathrm{~K}$ \\
Surface temperature 1 & $T_{g, 1}$ & 282.2 & 298 & $\mathrm{~K}$ \\
Surface temperature 2 & $T_{g, 2}$ & 260.7 & 277 & $\mathrm{~K}$ \\
Material entropy production & $\dot{S}_{\text {mat }}$ & 28.8 & 51 & $\mathrm{~mW} \mathrm{~m}^{-2} \mathrm{~K}^{-1}$ \\
Horizontal entropy production & $\dot{S}_{\text {hor }}$ & 7.5 & - & $\mathrm{mW} \mathrm{m}^{-2} \mathrm{~K}^{-1}$ \\
Vertical entropy production & $\dot{S}_{\text {ver }}$ & 21.2 & - & $\mathrm{mW} \mathrm{m}^{-2} \mathrm{~K}^{-1}$ \\
\hline
\end{tabular}

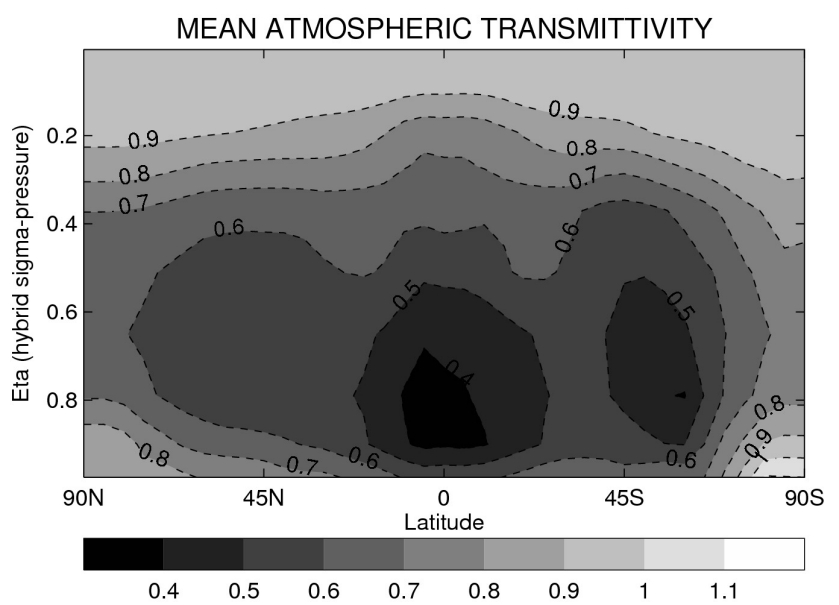

Fig. 6. Atmospheric transmissivity from a 30 -year mean FAMOUS control run.

\subsection{Radiative heating rates and entropy production}

Given the longwave transmissivities $\tau_{1}(\boldsymbol{x})$ and emissivities $\epsilon(\boldsymbol{x})$ as a function of 3-D position $\boldsymbol{x}=(x, y, z)$, the longwave heating rates $Q_{\mathrm{lw}}(\boldsymbol{x})$ can be obtained at every grid-point as a function of the temperature field. From Eq. (11) we have that for the top atmospheric layer $(z=N)$, $D(N-1 / 2)=\epsilon(N) \sigma T_{\mathrm{a}, N}^{4}$, since the downwelling longwave fluxes are null at the TOA (half level $N+1 / 2$ ). Hence by iteration all the downwelling longwave fluxes can be obtained for any temperature profile within the column. Likewise a similar iterative process is applied to obtain the upwelling fluxes $U$ by using equation in Eq. (10) once the lowest value is set $U(1 / 2)=\sigma T_{g}^{4}$. The knowledge of both upwelling and downwelling longwave fluxes for any given temperature field allows the calculation of the longwave heating rates as $Q_{\mathrm{lw}}=-\partial_{z}(U+D)$ and hence

$Q_{\mathrm{lw}}=Q_{\mathrm{lw}}[T(\boldsymbol{x})]$.
Adding the prescribed shortwave heating to $Q_{\mathrm{lw}}$, we obtain the net radiative heating:

$Q_{\mathrm{rad}}[T(\boldsymbol{x})]=Q_{\mathrm{sw}}(\boldsymbol{x})+Q_{\mathrm{lw}}[T(\boldsymbol{x})]$.

The material entropy production at this point can be expressed in terms of the radiative heating rates (as shown in Goody, 2000; Pascale et al., 2011a; Lucarini et al., 2011, also known as the inverse formula):

$\dot{S}_{\text {mat }}=-\int \frac{Q_{\text {rad }}}{T} d V$

(the integral is over the climate system and $T$ denotes $T_{\mathrm{a}}$ inside the atmosphere and $T_{\mathrm{S}}$ at the surface) provided that the climate system is in a steady state. This condition, for our model is, implies that the total radiative heating is null:

$\int Q_{\mathrm{rad}}[T(\boldsymbol{x})] d V=0$.

The validity of the inverse formula (based on radiative fields only) for expressing the material entropy production can be easily understood if we consider the direct formula (Eq. 9). For a steady state the terms $M-H_{1}, H_{2}-M, H_{1}$ and $H_{2}$ representing the material "diabatic" heating rates for each box of the model shown in Fig. 1 can alternatively be expressed in terms of the local radiative heating through the Eqs. (1)-(4) because material and radiative heat convergence have equal magnitude and opposite sign. If the system is not in a steady state the material entropy production is still expressed by Eq. (9) but no longer by the inverse formula.

From Eqs. (13)-(15) it is seen that in our model the material entropy production is defined as a positive-definite functional of the climate temperature field, i.e. $\dot{S}_{\text {mat }}=\dot{S}_{\text {mat }}[T(x)]$. Using $T(\boldsymbol{x})$ from FAMOUS, the value of the material entropy production we estimate is about $47 \mathrm{~mW} \mathrm{~m}^{-2} \mathrm{~K}^{-1}$, that is around $5 \mathrm{~mW} \mathrm{~m} \mathrm{~m}^{-2} \mathrm{~K}^{-1}$ less than the value diagnosed from FAMOUS in Pascale et al. (2011a). This is due to the the 

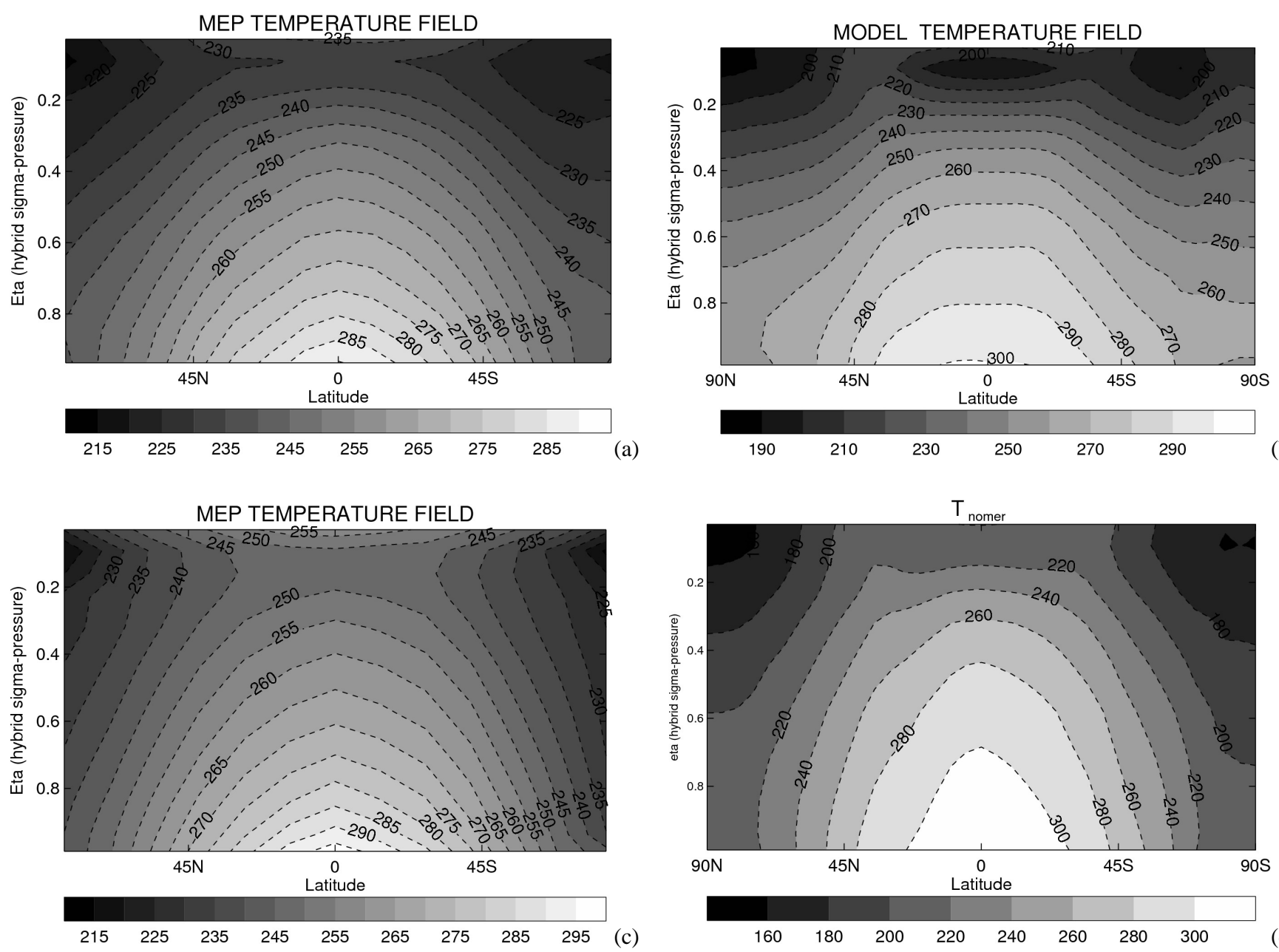

Fig. 7. (a) MEP temperature field as obtained from the maximisation problem discussed in Sect. 3.4. (b) FAMOUS temperature field. The values are obtained from a 30-year mean. (c) MEP2 maximising atmospheric temperature. (d) NOHH, no meridional heat transport.

fact that here we use time means $\overline{(\cdot)}$ for calculations and at the surface $\int \overline{Q_{\mathrm{sw}}} / \overline{T_{\mathrm{s}}}-\overline{\int Q_{\mathrm{sw}} / T_{\mathrm{s}}} \sim 5 \mathrm{~mW} \mathrm{~m}^{-2} \mathrm{~K}^{-1}$ in the FAMOUS climatology.

\subsection{MEP solution with prescribed transmissivity}

The Maximum Entropy Production conjecture applied to the model presented in this section can be formulated as a constrained variational problem (Noda and Tokioka, 1983; Schulman, 1977; Ito and Kleidon, 2005): the MEP temperature field is the one which maximises $\dot{S}_{\text {mat }}$ under the energy balance constraint (Eq. 16). For our model such a solution is defined as the field $T_{\mathrm{MEP}}$ such that:

$\frac{\delta \dot{S}_{\mathrm{mat}}}{\delta T}\left[T_{\mathrm{MEP}}\right]=0$ and $\int Q_{\mathrm{rad}}\left[T_{\mathrm{MEP}}\right] d V=0$,

where $\delta$ is the functional derivative and $V$ the volume of the climate system. We have assumed no surface net heat flux into the ocean and therefore radiative fluxes and material heat fluxes balance each other at the surface. A numerical solution is found by using the IDL 7.0 ${ }^{\circledR}$ (an array-orientated data analysis environment widely used in climate sciences) optimisation routine IMSL_CONSTRAINED_NLP (documentation available at http://idlastro.gsfc.nasa.gov/idl_html_help/ IMSL_CONSTRAINED_NLP.html), which can treat maximisation problems under non-linear constraints. Two numerical solutions, $T_{\mathrm{MEP} 1}$ and $T_{\mathrm{MEP} 2}$, are found for the $\epsilon=e / \sigma T^{4}$ and $\epsilon=1-\tau_{1}$ cases (Figs. 7a and 9), which in the following we will refer as MEP1 and MEP2 respectively. IMSL_CONSTRAINED_NLP accepts an optional initial guess from which the numerical search is started. Several different initial guesses are then used with IMSL_CONSTRAINED_NLP in order to verify the convergence of the numerical solution and exclude the possibility of multiple maxima. 


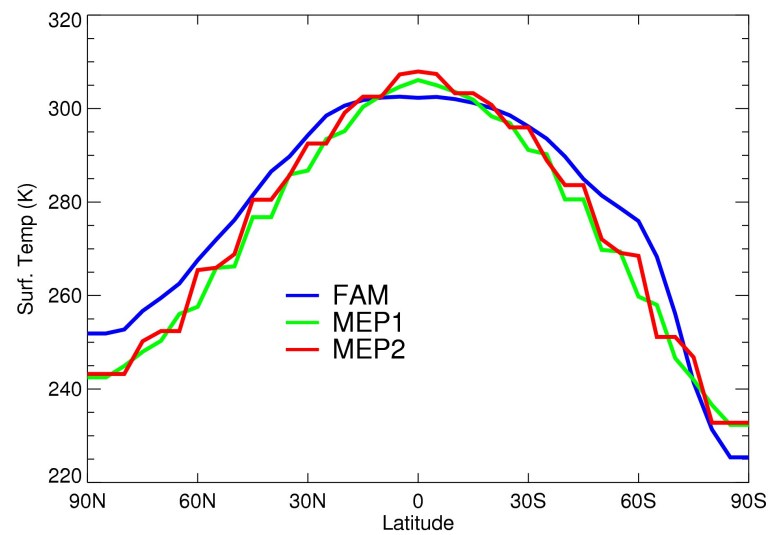

(a)

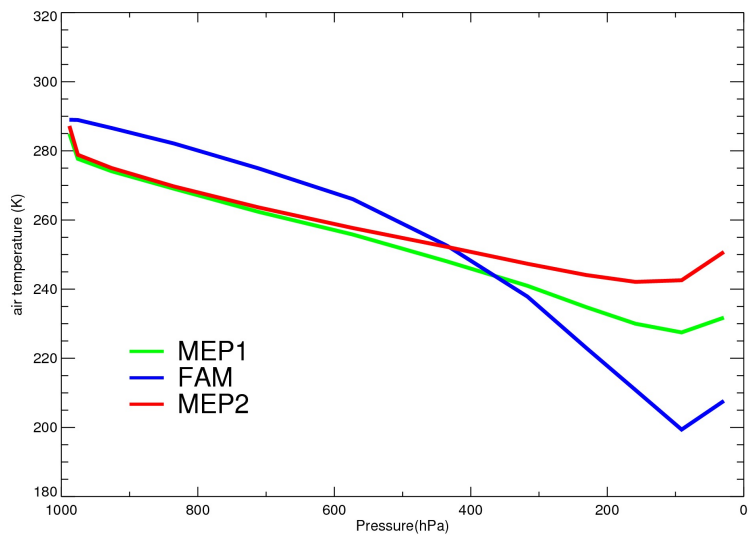

(c)

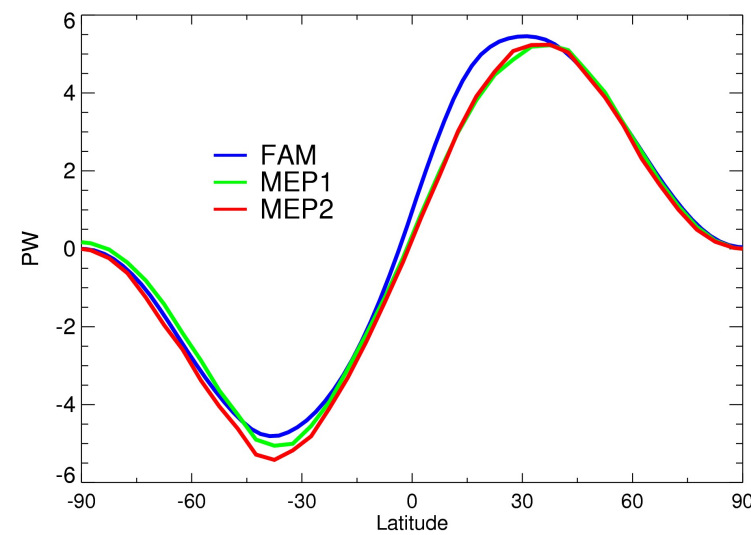

(b)

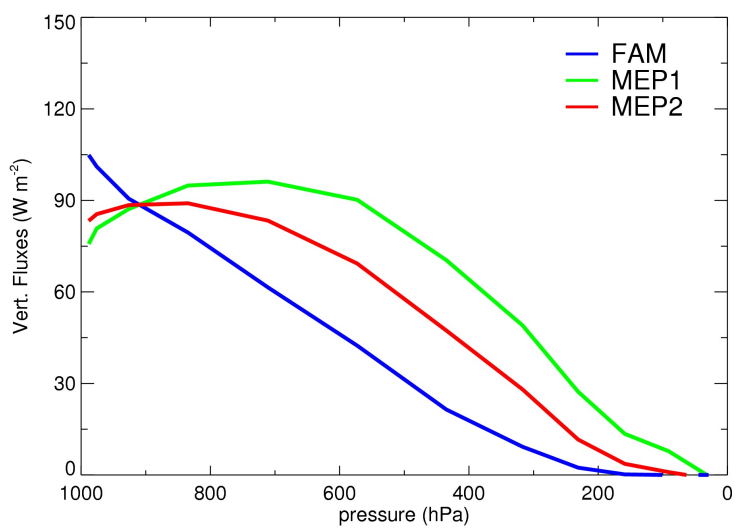

(d)

Fig. 8. Surface temperature (a), meridional heat transport (b), global mean temperature vertical profile (c) global mean vertical heat flux (d) for FAMOUS control run (FAM), MEP1 and MEP2.

\subsection{Comparison with the GCM solution}

The MEP1 and MEP2 solutions are compared with the FAMOUS climatology in Fig. 7b. It is undoubtedly fascinating how the MEP solutions resemble the FAMOUS one, particularly if we consider that this solution has involved no dynamics at all. The value of the material entropy production for MEP1 is $\dot{S}_{\text {mat }}\left[T_{\mathrm{MEP} 1}\right] \sim 70 \mathrm{~mW} \mathrm{~m}^{-2} \mathrm{~K}^{-1}$, which is larger than the FAMOUS one (about $50 \mathrm{~mW} \mathrm{~m}^{-2} \mathrm{~K}^{-1}$ ) and than any other value obtained from the different temperature fields examined in Sect. 4.2 (see Table 3). MEP2 has a material entropy production $\approx 57 \mathrm{~mW} \mathrm{~m}^{-2} \mathrm{~K}^{-1}$. The MEP1 and MEP2 fields related to the horizontal structure (i.e. surface temperature, atmospheric temperature, meridional heat transport) are fairly close to FAMOUS solution. This is true for the surface temperature field (Fig. 8a) and the meridional heat transport (Fig. 8b).

The main differences between the MEP solutions and the FAMOUS one are in the vertical structure. It can be seen that $T_{\mathrm{MEP} 1}$ and $T_{\mathrm{MEP} 2}$ tend to be warmer than $T_{\mathrm{FAM}}$ in the upper atmosphere and colder in the lower atmosphere. This feature is clearly seen in the global mean of the temperature profiles in Fig. 8c. The MEP solutions are more vertically mixed and reasonably in agreement with the one shown at p.443 of Ozawa and Ohmura (1997). The second remarkable difference is the discontinuity between surface temperature and near-surface atmospheric temperature, which in the MEP solutions is unrealistically large $(\approx 6 \mathrm{~K}$ whereas in FAMOUS it is $<1 \mathrm{~K}$ ). The MEP solution shows this feature also in Pujol and Fort (2002), who found a difference between the ground temperature and the air temperature at the surface $\sim 10 \mathrm{~K}$. Such unrealistic features of the vertical thermal structure (which turns out to be convectively unstable) may imply that some relevant physical constraints is missing in the model. For example Pujol (2003), in a one-dimensional radiative-convective climate model, shows that the inclusion of the temperature-opacity feedback significantly eliminates the convective instability of the vertical temperature profile 


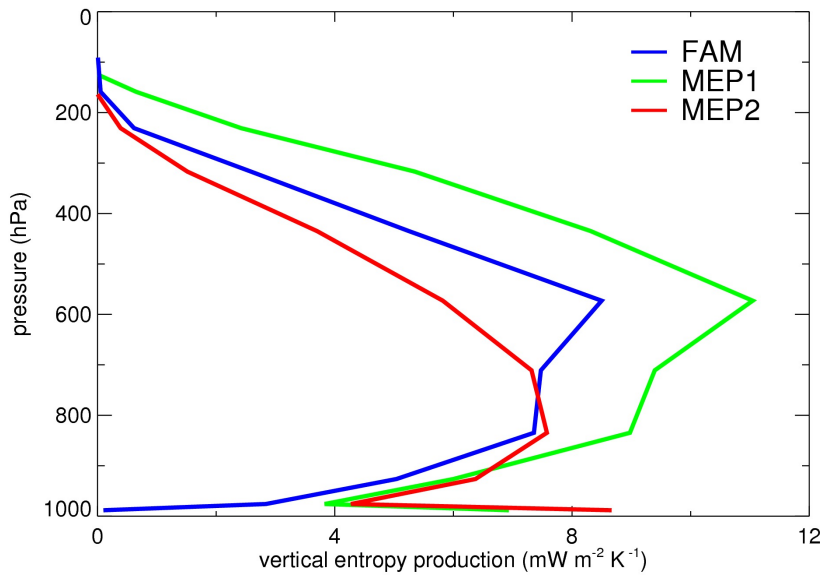

Fig. 9. Contributions to $\dot{S}_{\text {vert }}$ from each model level for FAMOUS control run (FAM), MEP1 and MEP2.

and the problem of the near-surface-to-ground temperature discontinuity.

The MEP solutions have a mean surface vertical heat flux of nearly $80 \mathrm{~W} \mathrm{~m}^{-2}$ (Fig. 8d), which is lower than the FAMOUS one $\left(\approx 100 \mathrm{~W} \mathrm{~m}^{-2}\right)$, and it is also lower in the two first atmosphere levels. Such a low value, which is in very good agreement with the one found by Pujol and Fort (2002) in their one dimensional model, explains the large discontinuity of the temperature at the surface. In the middle- and upper-atmosphere the mean vertical heat flux exceeds the one of the the FAMOUS solution. This means that a larger amount of heat is carried upwards which reduces the mean lapse rate and thus increases the atmospheric stability.

\subsection{Physical consistency of the MEP results}

To obtain the MEP solutions $T_{\mathrm{MEP}}$ in Sect. 3.5 we have assumed a fixed longwave transmissivity $\tau_{1}$ taken from a climatological mean of FAMOUS. However the local infrared optical depth depends on many variables, most importantly on the temperature, the concentration of water vapour and on cloud cover, and the latter two relate again to temperature and to other variables. As a consequence there must exist a climatically determined relation $\tau_{1}=\tau_{1}(T)$ which links longwave transmissivity to climate, where for convenience $T$ stands for all climatic variables. In any model to which we apply MEP the definition of $\tau_{1}(T)$ is therefore very important and a wrong definition of $\tau_{1}(T)$ will lead to the wrong set of permissible states on which MEP can operate, and the resulting $T_{\text {MEP }}$ will be incorrect.

For idealised climate models simple relations for $\tau_{1}(T)$ can be written down (e.g. Pujol, 2003), but for a realistic threedimensional climate it will be far more complex and $\tau_{1}(T)$ is unknown. In our case the MEP solutions $T_{\text {MEP }}$ shown in Sect. 3.4 have been found for $\tau_{\mathrm{FAM}}=\tau_{\mathrm{l}}\left(T_{\mathrm{FAM}}\right)$. Such a state $T_{\mathrm{MEP}}$ is not physically consistent with $T_{\mathrm{FAM}}$ because
Table 3. Entropy production summary for the seven temperature configurations. All entropy production in $\mathrm{mW} \mathrm{m}^{-2} \mathrm{~K}^{-1}$.

\begin{tabular}{lccc}
\hline climate & $\dot{S}_{\text {mat }}$ & $\dot{S}_{\text {vert }}$ & $\dot{S}_{\text {hor }}($ residual $)$ \\
\hline FAM & 47 & 40 & 7 \\
MEP1 & 70 & 62 & 8 \\
MEP2 & 57 & 46 & 11 \\
NOHT & 39 & 38 & 1 \\
NOHH & 41 & 41 & 0 \\
NOVT & 6 & 0 & 6 \\
\hline
\end{tabular}

generally $\tau_{1}\left(T_{\mathrm{MEP}}\right) \neq \tau_{\mathrm{FAM}}$. We speculate that the unrealistic features of $T_{\text {MEP }}$ (mainly in the vertical structure, see Fig. 8) may be due to the fact that we are not imposing the (unknown) relationship $\tau_{1}(T)$. By assuming a prescribed $\tau_{1}$ we have somehow assumed that $\tau_{1}(T)$ does not vary very much with temperature (zero-order approximation). In fact this is not true in FAMOUS, which embodies many physical constraints, without which the resulting solutions are physically unrealistic.

\subsection{MEP solution with variable transmissivity}

To examine further the consequences of this point, let us consider simultaneous independent variations of the longwave transmissivity $\tau_{1}$ and the temperature field $T$, and assume $\epsilon=1-\tau_{1}$. In this case the whole plane $\left(T, \tau_{1}\right)$ will be checked in the variational problem. Therefore under the constraint (Eq. 16) we look for $T$ and $\tau_{1}$ such that:

$\frac{\delta \dot{S}_{\mathrm{mat}}}{\delta T}\left[T_{\mathrm{MEP}}, \tau_{1, \mathrm{MEP}}\right]=0, \frac{\delta \dot{S}_{\mathrm{mat}}}{\delta \tau}\left[T_{\mathrm{MEP}}, \tau_{1, \mathrm{MEP}}\right]=0$.

A MEP solution (TAUTEMP) is obtained for a material entropy production within the range $[160,180]$ $\mathrm{mW} \mathrm{m} \mathrm{m}^{-2} \mathrm{~K}^{-1}$. The fields we obtain now are highly unrealistic. $\tau_{\mathrm{MEP}}$ tends to zero in the lower and middle atmosphere and sharply goes to unity in the uppermost atmosphere (Fig. 10a), which means that the atmosphere becomes completely opaque to the longwave radiation except in the upper atmosphere, thus shifting up the Earth's emission level (i.e. the level from which most of the TOA longwave radiation is emitted). The temperature is considerably higher than the one on Earth (Fig. 10b).

Unlike the MEP solution in Sect. 3.4, which is fairly well defined and insensitive to the initial guess, the MEP solutions found now seem to show a "weak" dependence on the initial guess, where by "weak" we mean here that different solutions differ for values of entropy production by $15 \%$ but show the same qualitative characteristics. This may also also mean that the algorithm is not converging. Therefore we will take them just as an indication of what happens when the constraint set for MEP is ill-posed. Furthermore, the fact that $\tau_{1, \mathrm{MEP}}$ is either zero or not zero (which are the bounds 


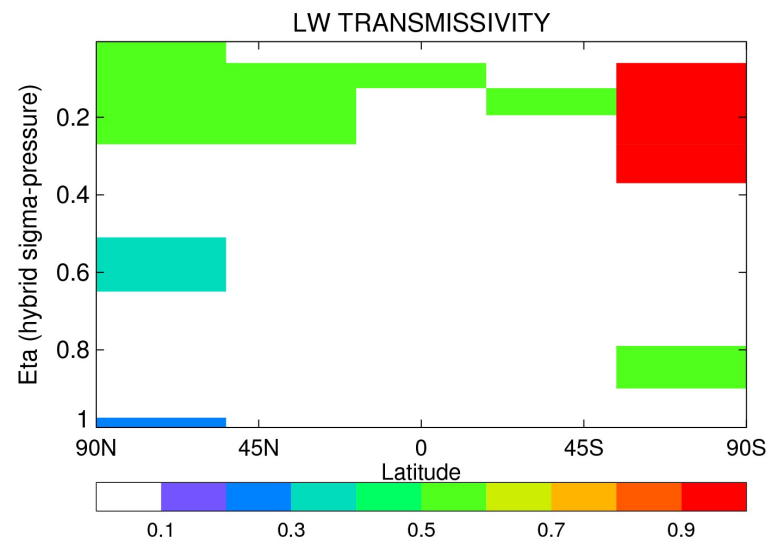

(a)

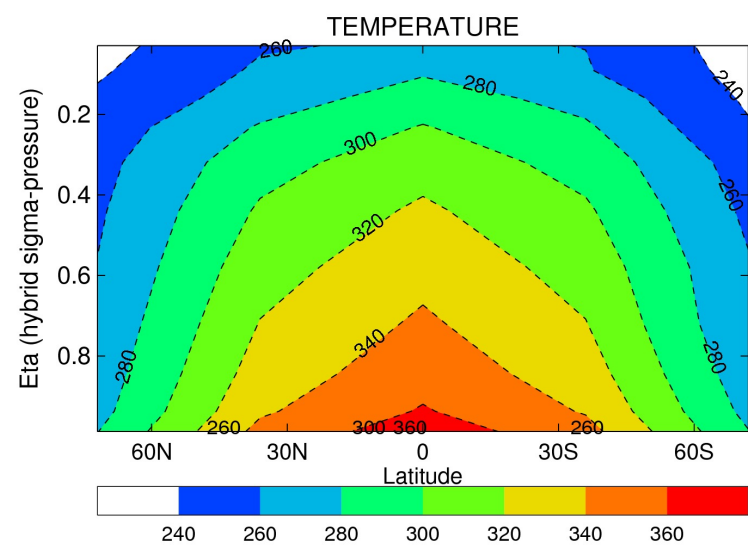

(b)

Fig. 10. LW transmissivity (a), atmospheric temperature (b) for the first TAUTEMP solution $\left(\dot{S}_{\text {mat }} \approx 160 \mathrm{~mW} \mathrm{~m}^{-2} \mathrm{~K}^{-1}\right)$.

for this variable) indicates that this is not a local maximum (i.e. inside the variables domain). We have checked that this is indeed the case in a simple three-box vertical model (two atmospheric boxes + surface) in which, for the sake of simplicity, we fix the temperatures and allow the two $\tau_{1}$ values to vary. The maximum of the material entropy production is achieved when the longwave transmissivity of the lower atmospheric box is equal to zero and that of the higher atmospheric box is about 0.65 , thus on the edge of the $\tau_{1}$ domain.

This substantial difference in the two solutions found under two different model formulations raises important scientific issue about the importance of the boundary conditions and the model formulation for the success of MEP (see also Goody, 2007). If the proper physical "ingredients" are not included in a low complexity climate model, the answer given by MEP is not realistic. This seems to agree with the interpretation of MEP given by Dewar (2009) or Dyke and Kleidon (2010), according to which MEP is an inference algorithm that passively translates physical assumptions into macroscopic predictions (as MaxEnt, Jaynes, 1957). In this respect in the variational problem which defines MEP the constraints assume the upmost importance. However often we do not know a priori what are the fundamental physical constraints that for a certain model have to be included or not, and this may restrict the practical application of MEP.

\section{Estimates of the vertical contribution to $\dot{S}_{\text {mat }}$}

\subsection{By averaging over horizontal dimensions}

In order to gain some qualitative understanding of the magnitude of the entropy produced by vertical heat transport we reduce the two-dimensional configurations to vertical onedimensional ones. This is obtained by averaging over the horizontal levels in order to eliminate the convergence of meridional heat transport. For an energy-balance model (no dynamics), given radiative, vertical and horizontal material heat fluxes $\mathbf{R}, \mathbf{H}, \mathbf{M}$, in a steady state we must have:

$\nabla \cdot \mathbf{R}+\nabla \cdot \mathbf{H}+\nabla \cdot \mathbf{M}=0$.

By defining the area-average as $\langle(\cdot)\rangle \equiv \int_{\Sigma}(\cdot) d \sigma / \int_{\Sigma} d \sigma$ and integrating over the $k$-th horizontal model level, of area $\Sigma_{k}$, we get rid of $\mathbf{M}$ :

$\int_{\Sigma_{k}} Q_{\mathrm{rad}, k} d \sigma+\int_{\Sigma_{k}} \nabla \cdot \mathbf{H} d \sigma=0$

because $\int_{\Sigma} \nabla \cdot \mathbf{M} d \sigma=0$ by definition over a horizontal surface. Therefore from Eq. (20) we can write $\langle\nabla \cdot \mathbf{H}\rangle=-\left\langle\partial_{z} H\right\rangle=-\left\langle Q_{\text {rad }}\right\rangle$. Given the mean flux at the surface, $\langle H\rangle_{1 / 2}$, and $\left\langle\partial_{z} H\right\rangle_{k}$, it is thus possible to work out $\left\langle H_{k+1 / 2}\right\rangle$ at every model half-level ( $Q_{\text {rad }}$ is defined on the full model levels, $k$ ). $\langle H\rangle$ (mass-weighted with the thickness of the model layer) is shown in Fig. 8d for the MEP and FAMOUS solutions. The material entropy production of the horizontally averaged vertical model is written as (Ozawa and Ohmura, 1997; Pujol and Fort, 2002):

$\dot{S}_{\mathrm{ver}}=\sum_{k}\left\langle H_{k+1 / 2}\right\rangle\left(\frac{1}{\left\langle T_{k+1}\right\rangle}-\frac{1}{\left\langle T_{k}\right\rangle}\right)$

in which $\left\langle T_{k}\right\rangle$ is simply the mean surface temperature at level $k$. The contribution of each model layer to $\dot{S}_{\mathrm{ver}}$, i.e. $\dot{S}_{\text {ver }, k}=\left\langle H_{k+1 / 2}\right\rangle\left(1 /\left\langle T_{k+1}\right\rangle-1 /\left\langle T_{k}\right\rangle\right)$, is shown in Fig. 9 for the FAM and the MEP solutions. As can be seen in Table $3, \dot{S}_{\text {ver }}$ is the term that really makes the difference between the MEP states and the FAMOUS climate (FAM).

Features to be pointed out are: (i) the first term $\dot{S}_{\text {ver, } 1 / 2}$ is substantially greater than zero $\left(\sim 7 \mathrm{~mW} \mathrm{~m}^{-2} \mathrm{~K}^{-1}\right)$ due to the notable surface-atmosphere discontinuity which is a feature of the MEP solution; (ii) contributions from the middleupper atmosphere remain significant, as shown in Fig. 9. 


\subsection{By constructing ad-hoc temperature fields}

In the previous section we have provided an order-ofmagnitude estimate of the $\dot{S}_{\text {ver }}$ and $\dot{S}_{\text {hor }}$. Based on an approximate equation derived from first principles Lucarini et al. (2011) recently found $\dot{S}_{\text {ver }} \approx 50 \mathrm{~mW} \mathrm{~m}^{-2} \mathrm{~K}^{-1}$ and $\dot{S}_{\text {hor }} \approx 5 \mathrm{~mW} \mathrm{~m}^{-2} \mathrm{~K}^{-1}$. Another independent estimate is provided in this section. This is obtained by examining some special significant temperature fields under the energy requirement expressed by Eq. (16). However, because of the fixed prescribed longwave optical properties (taken from FAMOUS climatology) which may not be fully consistent with the spatial temperature fields (see Sect. 3.7), we will take them more as an order-of-magnitude estimate rather than precise ones (or, put in different way, as a "zero"-order approximation since we are neglecting $\partial \tau_{1} / \partial T$ ).

First, let us consider $\dot{S}_{\text {ver }}$. If we consider a temperature field with no meridional temperature gradients (called NOHT, i.e. NO Horizontal Temperature gradient) then $\dot{S}_{\text {hor }}=0$ by construction and $\dot{S}_{\text {mat }}=\dot{S}_{\text {ver }}$. Such a field is obtained by replacing the temperature field from FAMOUS climatology with one which is horizontally uniform over each vertical level. The uniform value of the temperature over each model vertical level is obtained by taking its surface mean over the surface at that level. Note that this case is different from the one considered in Sect. 4.1: in the calculation involving $T_{\mathrm{NOHT}}$ we use the climatic longwave transmissivity field, which is not uniform horizontally (see Fig. 6), and therefore the radiative heating rate field will not be horizontally uniform, even though the temperature field is uniform. Second, we consider a temperature field $T_{\mathrm{NOHH}}$ which has the characteristic of producing a TOA longwave radiation equal at each point to the net incoming shortwave radiation, and therefore the net flux of radiation $F_{\text {rad }}^{\text {TOA }}$ equals zero at each point. Since the meridional divergence of the meridional heat transport in a steady state equals the net radiative flux at the TOA (Peixoto and Oort, 1992), zero TOA flux implies that $M$ is independent of latitude, which means it must be zero at every latitude since it vanishes at the poles. The temperature field is obtained as $T_{\mathrm{NOHH}}=\alpha^{1 / 4} T_{\mathrm{FAM}}$, where $\alpha=\mathrm{SW} / \mathrm{LW}$ is the ratio between the magnitude of the net shortwave and longwave fluxes at the top of the atmosphere from the FAMOUS climatology (Fig. 7d). In fact if we assume heuristically $\mathrm{LW}_{\mathrm{NOHH}} \sim \sigma T_{\mathrm{NOHH}}^{4}$, then $\mathrm{LW}_{\mathrm{NOHH}} \sim \mathrm{SW}$. By using our approximations we find $\dot{S}_{\text {mat }} \approx 39 \mathrm{~mW} \mathrm{~m}^{-2} \mathrm{~K}^{-1}$ and $\dot{S}_{\text {mat }} \approx 41 \mathrm{~mW} \mathrm{~m}^{-2} \mathrm{~K}^{-1}$ for $T_{\mathrm{NOHT}}$ and $T_{\mathrm{NOHH}}$ respectively (Table 3). These values are of the same order of magnitude of the estimate of $\dot{S}_{\mathrm{ver}}$ obtained for $T_{\mathrm{FAM}}$ in Sect. 4.1.

Second, let us consider a temperature field $T_{\text {NOVT }}$ which is vertically homogeneous (NOVT, NO Vertical Temperature gradient): the temperature is constant throughout each vertical column $\left(\partial_{z} T=0\right.$, including the surface as well) but with a meridional gradient. Such a field is defined as $T_{\mathrm{NOVT}}=\int \rho d z T_{\mathrm{FAM}} / \int \rho d z-24 \mathrm{~K}$ (where the $-24 \mathrm{~K}$ degree is needed to satisfy the energy balance, Eq. 16). As a consequence $\dot{S}_{\text {mat }} \approx \dot{S}_{\text {mat }}^{\text {hor }}$. We find that $\dot{S}_{\text {mat }} \approx 6 \mathrm{~mW} \mathrm{~m}^{-2} \mathrm{~K}^{-1}$, which is of the right order of magnitude if compared with the estimate obtained in Sect. 4.1 (see Table 3) for $T_{\text {FAM }}$.

Our estimates of $\dot{S}_{\text {ver }}$ and $\dot{S}_{\text {hor }}$, although based on a crude method, are quite close to those obtained in Sect. 4.1 and by Lucarini et al. (2011).

\section{Conclusions}

This paper provides insights into the application of MEP to a simple four-box model of climate able to represent both horizontal and vertical heat fluxes and temperature gradients, which are the major characteristics of our climate system. By keeping insolation and optical properties of each atmospheric box fixed (i.e. the surface albedo, shortwave and longwave transmissivity), a MEP solution can be found with numerical values of temperatures and heat fluxes reasonably realistic given the simplicity of the model. These results extend the two-box analysis of Lorenz et al. (2001) and Kleidon (2009) and address the issues raised by Lucarini et al. (2011) regarding the fact that both vertical and horizontal processes contributing to the material entropy production have to be included. The analysis of the "horizontal" $\dot{S}_{\text {hor }}$ and "vertical" $\dot{S}_{\text {ver }}$ component of the material entropy production shows that $\dot{S}_{\text {hor }}$ is almost independent of the vertical heat fluxes. Since $\dot{S}_{\text {hor }}$ is entirely due to atmospheric processes, this result is consistent with the maximum power conjecture discussed in Kleidon (2010) in terms of a two-box model (as the mechanical power dissipated by the climate system is almost entirely associated with the atmospheric circulation). Also, $\dot{S}_{\text {ver }}$ is independent of the horizontal heat flux $M$ i.e. horizontal and vertical material entropy production are independent.

Further insight is obtained by considering a zonal-vertical model of climate analogous to the four-box model but with increased resolution. Radiative parameters (shortwave and longwave transmissivity) are derived from a GCM climatology. A MEP solution is found showing a surprising realism as far as the surface temperature and transport of meridional heat are concerned but major discrepancies with FAMOUS climatology (assumed as representative of real climate, see Jones et al., 2005) are found in the vertical organisation of the atmosphere and in an unrealistic surface-atmosphere temperature discontinuity $(\approx 6 \mathrm{~K})$. This study therefore suggests that the prediction of vertical convection requires different essential physical constraints than horizontal transport. By using an averaging technique and ad hoc temperature configurations we obtain an order-of-magnitude decomposition of the total material entropy production $\left(\approx 50 \mathrm{~mW} \mathrm{~m}^{-2} \mathrm{~K}^{-1}\right)$ into its horizontal component $\dot{S}_{\text {hor }} \approx 5-7 \mathrm{~mW} \mathrm{~m}^{-2} \mathrm{~K}^{-1}$ and its vertical component $\dot{S}_{\text {ver }} \approx 40 \mathrm{~mW} \mathrm{~m}^{-2} \mathrm{~K}^{-1}$ which agrees with independent estimates given by Lucarini et al. (2011) using an approximate formula derived from the general equations of entropy balance. 
Acknowledgements. S. P. acknowledge the financial support of the Reading International studentship and EU-ERC research grant NAMASTE. V. L. also acknowledges the NAMASTE grant. J. M. G. acknowledges support from the NCAS-Climate programme. The authors thank the four anonimous referees for their helpful comments.

Edited by: A. Kleidon

\section{References}

Ambaum, M. H. P.: Thermal physics of the atmosphere, Wiley-Blackwell, ISBN 978-0-470-74515-1, eBoook: doi:10.1002/9780470710364, 256 pp., Chichester, 2010.

Busse, F.: On Howard's upper bound for heat transport by thermal convection, J. Fluid Mech., 37, 457-477, 1969.

Busse, F. H.: Bounds for turbulent shear flow, J. Fluid Mech., 41, 219-240, 1970.

Caldeira, K.: The maximum entropy principle: a critical discussion, Climatic Change, 85, 267-269, 2007.

DeGroot, S. and Mazur, P.: Non-equilibrium thermodynamics, Dover, 1984.

Dewar, R. C.: Maximum entropy production and the fluctuation theorem, J. Phys. A, 38, L371-L381, 2005.

Dewar, R. C.: Maximum entropy production as an inference algorithm that translates physical assumption into macroscopic predictions: don't shoot the messenger, Entropy, 11, 931-944, 2009.

Dufresne, J., Fournier, R., Hourdin, C., and Hourdin, F.: Net exchange reformulation of radiative transfer in the $\mathrm{CO}_{2} 15 \mu \mathrm{m}$ band on Mars, J. Atmos. Sci., 62, 3303-3319, 2005

Dyke, J. and Kleidon, A.: The maximum entropy production principle: its theoretical foundations and applications to the earth system, Entropy, 12, 613-630, 2010.

Edwards, J. and Slingo, A.: Studies with a flexible new radiation code, Part one: Choosing a configuration for a large-scale model, Q. J. Roy. Meteorol. Soc., 122, 689-719, 1996.

Fraedrich, K. and Lunkeit, F.: Diagnosing the entropy budget of a climate model, Tellus A, 60, 921-931, 2008.

Goody, R.: Sources and sinks of climate entropy, Q. J. Roy. Meteorol. Soc., 126, 1953-1970, 2000.

Goody, R.: Maximum entropy production in climate theory, J. Atmos. Sci., 64, 2735-2739, 2007.

Gordon, C., Cooper, C., Senior, C., Banks, H., Gregory, J., Johns, T., Mitchell, J., and Wood, R. A.: The simulation of SST, sea ice extents and ocean heat transports in a version of the Hadley Centre coupled model without flux adjustments, Clim. Dynam., 16, 147-168, 2000.

Grassl, H.: The climate at the maximum-entropy production by meridional atmospheric and oceanic heat fluxes, Q. J. Roy. Meteorol. Soc., 107, 153-166, 1981.

Grassl, H.: Foreword, in: Non-equilibrium thermodynamics and the production of entropy, Springer, 2005.

Grinstein, G. and Linsker, R.: Comments on a derivation and application of the maximum entropy production principle, J. Phys. A, 40, 9717-9720, 2007.

Herbert, C., Paillard, D., and Dubrulle, B.: Entropy production and multiple equilibria: the case of the ice-albedo feedback, Earth Syst. Dynam., 2, 13-23, doi:10.5194/esd-2-13-2011, 2011 a.
Herbert, C., Paillard, D., Kageyama, M., and Dubrulle, B.: Present and last glacial maximum climates as states of maximum entropy production, Q. J. Roy. Meteorol. Soc., 137, 1059-1069, 2011b.

Ito, T. and Kleidon, A.: Non-equilibrium thermodynamics and the production of entropy, chapter 8, Entropy production of atmospheric heat transport, pages, Springer, 93-106, 2005.

Jaynes, E.: Information theory and statistical mechanics, Phys. Rev., 106, 620-630, 1957.

Jones, C., Gregory, J., Thorpe, R., Cox, P., Murphy, J., Sexton, D., and Valdes, P.: Systematic optimisation and climate simulation of FAMOUS, a fast version of HadCM3, Clim. Dynam., 25, 189204, 2005.

Jupp, T. and Cox, P.: MEP and planetary climates: insights from a two-box climate model containing atmospheric dynamics, Philos. T. Roy. Soc. B, 365, 1355-1365, 2010.

Kleidon, A.: Beyond gaia: thermodynamic of life and earth system functioning, Climatic Change, 66, 271-319, 2004.

Kleidon, A.: Nonequilibrium thermodynamics and maximum entropy production in the earth system, Naturwissenschaften, 96, 653-677, 2009.

Kleidon, A.: A basic introduction to the thermodynamics of the earth system far from equilibrium and maximum entropy production, Philos. T. Roy. Soc. B, 365, 1303-1315, 2010.

Kleidon, A., Fraedrich, K., and Kunz, T., and Lunkeit, F.: The atmospheric circulation and the states of maximum entropy production, Geophys. Res. Lett., 30, 2223, doi:10.1029/2003GL018363, 2003.

Kleidon, A., Fraedrich, K., Kirk, E., and Lunkeit, F.: Maximum entropy production and the strenght of boundary layer exchange in an atmospheric general circulation model, Geophys. Res. Lett., 33, L08709, doi:10.1029/2005GL025373, 2006.

Kunz, T., Fraedrich, K., and Kirk, E.: Optimisation of simplified GCMs using circulation indices and maximum entropy production, Clim. Dynam., 30, 803-813, 2008.

Lorenz, E.: Generation of available potential energy and the intensity of the general circulation, Pergamon, Tarrytown, N.Y., 1960.

Lorenz, R., Lunine, J., Withers, P., and McKay, C.: Titan,Mars and Earth: Entropy production by latitudinal heat transport, Geophys. Res. Lett., 28, 415-418, 2001.

Lucarini, V.: Thermodynamic efficiency and entropy production in the climate system, Phys. Rev. E, 80, 021118, doi:10.1103/PhysRevE.80.02118, 2009.

Lucarini, V., Fraedrich, K., and Ragone, F.: New results on the thermodynamic properties of the climate, J. Atmos. Sci., 68, 24382458, 2011.

Malkus, W.: Outline of a theory of turbulent shear flow, J. Fluid Mech., 1, 521-539, 1956.

Malkus, W.: Borders of disordrs: in turbulent channel flow, J. Fluid Mech., 489, 185-198, 2003.

Malkus, W. V. R.: The heat transport and spectrum of thermal turbulence, P. Roy. Soc. Lond. A, 225, 196-212, 1954.

Martyushev, L. and Seleznev, V.: Maximum entropy production principle in physics, chemistry and biology, Phys. Rep., 426, 145, 2006.

Murakami, S. and Kitoh, A.: Euler-Lagrange equation of the most simple 1-d climate model based on the maximum entropy production hypothesys, Q. J. Roy. Meteorol. Soc., 131, 1529-1538, 2005. 
Nicolis, C. and Nicolis, G.: Stability, complexity and the maximum dissipation conjecture, Q. J. Roy. Meteorol. Soc., 136, 11611169, 2010.

Noda, A. and Tokioka, T.: Climates at minima of the entropy exchange rate, J. Meteorol. Soc. Jpn., 61, 894-908, 1983.

Ozawa, H. and Ohmura, A.: Thermodynamics of a global-mean state of the atmosphere: A state of maximum entropy increase, J. Climate, 10, 441-445, 1997.

Ozawa, H., Ohmura, A., Lorenz, R., and Pujol, T.: The second law of thermodynamics and the global climate system: a review of the maximum entropy production principle, Rev. Geophys., 41, 1018, doi:10.1029/2002RG000113, 2003.

Paltridge, G.: Thermodynamic dissipation and the global climate system. Q. J. Roy. Meteorol. Soc., 107, 531-547, 1981.

Paltridge, G. W.: Global dynamics and climate-a system of minimum entropy exchange, Q. J. Roy. Meteorol. Soc., 101, 475-484, 1975.

Paltridge, G. W.: The steady state format of global climate, Q. J. Roy. Meteorol. Soc., 104, 927-945, 1978.

Pascale, S., Gregory, J., Ambaum, M., and Tailleux, R.: Climate entropy budget of the HadCM3 atmosphere-ocean general circulation model and FAMOUS, its low-resolution version, Clim. Dynam., 36, 1189-1206, 2011a.

Pascale, S., Gregory, J., Ambaum, M., and Tailleux, R.: A parametric sensitivity study of entropy production and kinetic energy dissipation using the FAMOUS AOGCM, Clim. Dynam., doi 10.1007/s00382-011-0996-2, in press, 2011b.
Peixoto, J. P. and Oort, A.: Physics of the Climate, Springer-Verlag, New York, 1992.

Pope, V. D., Gallani, M. L., Rowntree, P. R., and Stratton, R. A.: The impact of new physical parametrizations in the Hadley Centre climate model - HadAM3, Clim. Dynam., 16, 123-146, 2000.

Pujol, T.: Eddy heat diffusivity at maximum dissipation in a radiative-convective one-dimensional climate model, J. Meteorol. Soc. Jpn., 81, 305-315, 2003.

Pujol, T. and Fort, J.: States of maximum entropy production in a one-dimensional vertical model with convective adjustments, Tellus A, 54, 363-369, 2002.

Reichler, T. and Kim, J.: How well do coupled models simulate today's climate? B. Am. Meteorol. Soc., 89, 303-311, 2008.

Rodgers, C.: Minimum entropy exchange principle-reply, Q. J. Roy. Meteorol. Soc., 102, 455-457, 1976.

Schulman, L. L.: A theoretical study of the efficiency of the general circulation, J. Atmos. Sci., 34, 559-580, 1977.

Shimokawa, S. and Ozawa, H.: On the thermodynamics of the oceanic general circulation: entropy increase rate of an open dissipative system and its surroundings, Tellus A, 53A, 266-277, 2001.

Smith, R. S., Gregory, J. M., and Osprey, A.: A description of the FAMOUS (version XDBUA) climate model and control run, Geosci. Model Dev., 1, 53-68, doi:10.5194/gmd-1-53-2008, 2008.

Trenberth, K., Fasullo, J., and Kiehl, J.: Earth's global energy budget, B. Am. Meteorol. Soc., 90, 311-324, 2009. 